\title{
Mathematical Model of Economical Assessment of Investments in Information Provision for the Management System of a Modern Company
}

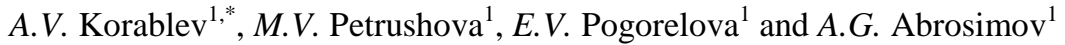 \\ *Corresponding author: korablyov.av@gmail.com \\ ${ }^{1}$ Samara State University of Economics, Samara, Russia
}

\begin{abstract}
Information provision for a company's management system not only provides data for evaluating day-to-day operations but also is an efficient tool for improving the reliability of the entire management system. For purposes of efficiently managing projects for implementing modern information provision, the company should design a formalized model for assessing the relationship between projectrelated financial costs and the number of automated business processes in place at the company. This paper proposes using a mathematical model that contains financial indicators such as net present value, cash flows, and discount rates. Thanks to lower investment risks, the model will improve the economic efficiency of investment projects as part of implementing information provision at the company.
\end{abstract}

Keywords: management system, information provision, information flow, performance indicator.

\section{Introduction}

Information provision for the management of a modern company involves the acceleration and rational movement of information flows, resulting in reduced time and financial and labor costs incurred in discharging management functions. That provision integrates the structure that comprises equipment, techniques, and staff. The integration is based on information support for monitoring, decision analysis, and strategic and operating planning.

\section{Problem Statement}

Principal problems hindering efficient implementation relate to human error and involve restructuring work organization. The latter problem is solvable by providing tools based on corporate management, including staff training and adaptation to new information environments.

Efficient information provision for the management system enables the company to achieve conditions for rapid qualitative and quantitative market growth. It is this that allows the company to preserve the necessary flexibility while keeping strategic and operating control as well as to ensure that client service steadily improves (an increase in the client base leads to more transactions, thereby improving profitability).

An information flow in the provision of information for the management system is an organized data movement from source to recipient. Information flows have distinctive features based on the properties of the information management system [1]. The efficiency of using an information flow in the system is assessable with a ratio of information used once to the total amount of required information and with a ratio of required information to the total amount of information used.

Managing an information flow consists in minimizing the rate at which information is collected and transmitted so that that rate is comparable with the rate at which information is received and processed as well as in minimizing the volume of the information flow consistent with the technical nuances of the route developed to direct the flow from source to recipient [2]. Aggregate financial costs should also be considered that relate to forming, transmitting, processing, and storing information flows (data). These costs are part of total information-related expenditure of the management system.

\section{Research Questions}

The following indicators characterize information flows: data flow capacity (the aggregate amount of information processed in a precise period); flow quality for a specific capacity (the accuracy degree of sent data); and the flow's total financial losses (total costs comprising those for transmitting data in the flow with specific quality and capacity parameters).

The processing of initial information about the state of the entity managed runs under the influence of governing effects that depend on the objectives pursued in managing the entity and on criteria for optimal management. The 
processing mechanism is based on information delivery systems. For purposes of studying information flows as exposed to external effects, the term document flow is used.

Using the nuances of managerial information, we can formulate criteria for developing the provision of information for the management system: creating a powerful input channel to receive documents; making stringent rules for routing documents; and developing security policies that meet modern requirements for data security.

The subsystem underlying information provision is a complicated organizational structure that consists of many functions. We will note that according to the methodology of the system approach, the composition of an information system matches the definition of a complex system [3]. Standardized documentation systems and classifications are used to organize information files. Those are used to create files that form information databases following a single technique [4].

An indicator that shows the quality degree of the information system in place and takes into account the identified operating costs can serve as an economic performance indicator. From the perspective of end-user satisfaction, this indicator can be used to determine the quality of the service in question.

\section{Purpose of the Study}

The purposes of determining the efficiency of the information system in place are as follows: setting the objectives of system operation; identifying the degree of interaction between system components; identifying financial cost items; establishing indicators to characterize the efficiency of information management; and selecting an assessment scale for the system performance indicator.

To calculate the performance indicator for the information system, one needs to assemble a group of indicators that show the state of the information system; identify critical values for each indicator with due regard to the assessment scale; develop software or an additional module for the existing corporate information system to use in practice the performance indicators of the information system; and design a method for evaluating the obtained results [5].

Developing an information system for service management involves the use of methods for complex management of integrated information flows that have an effect on how quickly managerial decisions are made and on financial costs related to information movement, which ultimately influences service quality [6].

Each information-flow operation has its own costs. Cost management is based on imposing a group of limitations on the information model as part of determining the target function. We will use quantitative characteristics of resources and assigned effectiveness values for top-level business processes as limitations imposed on the target function.

This allows us to determine that the integral criterion for the optimality of total costs is an assessment criterion for how efficiently the information system operates [7,8]. Let us write the criterion as

$$
E f=\sum_{i}^{\mathrm{p}} \sum_{j}^{f} \sum_{k}^{z} \mathrm{Qijk}-\sum C
$$

where $Q i j k$ is the value of services provided for the $i$ th operation of the $j$ th function of the $k$ th process; $C$ is the value of total costs for the $i$ th operation of the $j$ th function of the $k$ th process; $p$ is the number of operations for the information flow; $f$ is the number of functions; $z$ is the number of business units involved in the transmission and processing of information.

\section{Research Methods}

Optimizing the movement of information flows consists in calculating quantitative values for an informationfunction model and calculating resources used for its operation. This also includes calculating the costs of altering, updating, removing, and designing operations that comprise certain management functions. To determine the final value for financial indicators of optimized flow processes as part of managing their operations, one needs to make an aggregate model for monitoring financial costs. For that purpose, we need to determine several factors that affect those costs. These factors are assessable according to their significance.

Factors affecting the reduction of financial, labor, and time costs as part of updating flow processes and developing information systems are the exclusion of functions that implement data entry operations when any data are redundant; the exclusion of control functions in implementing a single integrated information system; the exclusion of document approval functions when the document is transmitted under routing rules; reducing costs when preparing analytical data (all source documents are available, and the time spent retrieving them is minimized); and reducing the cost of fulfilling control functions when using sequential automation.

Factors affecting an increase in financial, labor, and time costs in implementing a project to develop an information system are an increase in costs incurred in collecting information when many details have to be entered to make the document more informative; costs incurred in training the company's staff in new advanced functions for working with the information system; and higher costs required for the technical, methodological, and software support of a new type of information system. 
To assess how effectively the information system operates, we will use the following model:

$$
\Delta R=p N P V(d, D)+M * N P V(d, P, C),
$$

where $\Delta R$ is the final present value of the cash flow that occurs in parallel to the movement of the information flow that ensures service provision; $p$ is the probability of the project being implemented successfully; $N P V$ is the net present value; $D$ is the amount of the cash flow that characterizes the life cycle of the information system; $P$ is the magnitude of probable financial costs involved in implementing the project; $C$ represents the investment costs of implementing the system; $d$ is a discount factor; and $M$ is the forecast expectation for present value if the project implementing the system is discontinued.

The variable $C$ characterizes the financial-cost flow. A feature of financial costs is that they are divided in time, resulting in net present value being recalculated in each specific instance [9]. Because of this, if the project implementing the information system is financially successful, the net present value of cash flows $D$ and $C$ - namely their sum - characterizes the factors that affect project implementation. Ultimately these factors have an effect on performance.

\section{Findings}

Optimizing the information system for service management requires considering two types of costs:

- $\quad$ principal costs (financial expenses for buying equipment and software; organizational expenses for managing IT departments; general expenses for developing, implementing, and maintaining the company's information system);

- indirect costs (losses caused by system downtime (productivity losses caused by dysfunctional software, including lost time); losses caused by entering wrong information; costs related to user support; and costs of creating an information security system).

A nuance of these systems is the problem of assessing final financial results. Those results depend on the uncertainty of cash flows, and so performance depends directly on steps taken to effectively modernize the improvement of flowbased service processes.

It is noteworthy that flow operations related to maintaining cash flows and cash-flow planning are interrelated as far as types of data are concerned; therefore, those operations should be streamlined efficiently.

The economic efficiency of running a project to implement an information system consists of two components: minimizing losses involved in running processes; increasing the rate of return on capital.

\section{Conclusion}

The economic efficiency of a project depends on the joint maximization of income as part of operating an information system and on the high probability of completing a project to implement that system with due regard to the mathematical expectation of losses caused by discontinuing the project. Because of this, improving financial performance should involve considering all possible options for running the implementation project, and the option to be selected is the one that yields the maximal cash flow. Steps to implement the project should be optimized to keep to a minimum the probability of the project being discontinued at a final implementation stage.

Information provision for the management system not only provides data for evaluating day-to-day operations but also is an efficient tool for improving the reliability of the entire management system. In identifying prospective development trends for the company, the access to external market information underpinning strategic decisions is limited.

To create an efficient information system for managing various data (regulations, standards, statistics, accounting entries, analytical reports) and techniques (for collecting, storing, transmitting, and processing information), one needs to simultaneously solve a variety of problems. Those problems may include both research in related fields (management, economics, and statistics) and application of modern information technology.

\section{References}

1. L. Galbusera, G. Giannopoulos, On input-output economic models in disaster impact assessment. International Journal of Disaster Risk Reduction, 30(B), 186-198. DOI: 10.1016/j.ijdrr.2018.04.030 (2018).

2. J. Luo, Z. Hu, Risk paradigm and risk evaluation of farmers cooperatives' technology innovation. Economic Modelling, 44(C), 80-85. DOI: 10.1016/j.econmod.2014.10.024 (2015).

3. P.P. Groumpos, Modelling Business and Management Systems Using Fuzzy Cognitive Maps: A Critical Overview. IFAC-Papers On Line, 48(24), 207-212. DOI: 10.1007/978-3-540-24581-0_22 (2015). 
4. G. Alfonso, A.F. Roldán López de Hierro, C.Roldán, A fuzzy regression model based on finite fuzzy numbers and its application to real-world financial data. Journal of Computational and Applied Mathematics, 318, 47-58. DOI: 10.1016/j.cam.2016.12.001 (2017).

5. A.P. Henriquesde, L. Camara, M. Mendonca, T. Poleto, Information security risk analysis model using fuzzy decision theory. International Journal of Information Management, 36(1), 25-34. DOI: 10.1016/j.ijinfomgt.2015.09.003 (2016).

6. M.I. Geras'kin, A.G. Chkhartishvili Structural modeling of oligopoly market under the nonlinear functions of demand and agents' costs. Automation and Remote Control, 78, 332-348. DOI: 10.1134/S0005117918050065 (2017). [in Rus.].

7. A.V. Korablev, Application of cloud technologies in banking activities. Journal of Economy and Entrepreneurship, 73, 463-468 (2016). [in Rus.].

8. M.F. Masneva, M.V.Petrushova, Internet portals of remote public services. Journal of Economy and Entrepreneurship, 67, 130-133 (2016). [in Rus.].

9. M.I. Geras'kin, Modeling reflexion in the non-linear model of the stakelberg three-agent oligopoly for the Russian telecommunication market. Automation and Remote Control, 79, 841-859. DOI: 10.1134/S0005117917020114. (2018). [in Rus.] 